and note the instantaneous effect that this produces on the Blind Worm. The little grey slug is a biologically relevant stimulus, since in the ordinary way Limax agrestis forms the elected article of diet of Anguis fragilis." Now what the blind-worm perceives is not a slug, as we perceive and recognize it, but merely something of a particular size, shape and mode of motion, which has food-valence. We must carefully guard against the fallacy of assuming that animals perceive objects in the detailed and complex way that we do, as continuing 'things', recognizable by a large number of associated characteristics ; the evidence tends to show, on the contrary, that animals may attend to and perceive only one or few characters of objects. That is why their perception sometimes leads them astray.

Many of the so-called aberrations of instinctive behaviour are quite understandable when we realize these two things: (1) that the animal's sensory or perceptual field is often a very simple one, and (2) that in normal conditions the events and the objects possessing valence are usually objects and events that are biologically significant. 'Aberrations' occur when, in unusual circumstances or by exception, the valent characteristic or clue crops up as a character of a biologically unsuitable object. But the animal does not necessarily confuse the two different perceptual objectsthe suitable and the unsuitable; it may perceive only the valent characteristic which is common to both, and fail to perceive the differentia of the two.
Within the limits of its instinctive perception, then, it does not err or make a mistake ; it is misled.

The fact that the sensory or perceptual field is usually a fairly simple one helps in some measure to reduce the mystery of instinctive behaviour; we have no need to assume that an animal is attuned by heredity to recognize, without prior experience, a highly complex and specific object, such as it appears to us.

${ }^{1}$ Cameron, E., "A Study of the Natural Control of the Pea Moth Cydia nigricana, Steph.", Bull. Entom. Research, 29, 277-313 (1938)

'Spaier, A., "De la nature de l'instinct", Revue philos., 55, 410-445 (1930).

"Ullyett, G. C., "Host Selection by Microplectron fuscipennis", Proc Roy. Soc., B, 120, 253-291 (1936).

- Russell, E. S., "Fish Migrations", Biol. Rev., 12, 320-337 (1937).

${ }^{3}$ Carpenter, G. D. H., "Some Notes on the Northern Islands of Lake Victoria", J. Animal Ecol., 111, 91-104 (1934).

"Doflein, F., "Der Ameisenlöwe", (Jena, 1916).

"Andrews, E. A., "The Seventeen Year Locust, alias Cicada”, Quart. Rev. Biol., 12, 271-293 (1937).

' Myers, J. G., "Insect Singers"' (London, 1929)

- Snodgrass, R. E., "The Seventeen Year Locust", Rep. Smithsonian Inst. for 1919, 408 (1921).

${ }^{10}$ Tillyard, R. J., "The Biology of Dragonflies" (Cambridge, 1917).

11 Hering, M., "Biologie der Schmetterlinge" (Berlin, 1926).

12 Thompson, W. R., and Parker, H. L., "Host Selection in Pyrausto nubilalis", Bull. Ent. Res., 18, 359-64 (1928).

${ }^{13}$ Barrows, W. M., "The Reactions of the Pomace Fly, Drosophile ampelophila, to Odorous Substances", J. Exp. Zool., 4 (1907).

14 Ilse, D., NATURE, 140, 544 (1937).

${ }^{15}$ Bodenheimer, F. S., “Problems of Animal Ecology"' (Oxford, 1938).

${ }^{16}$ Lloyd, D. C., "A Study of some Factors governing the Choice of Hosts and Distribution of Progeny by the Chalcid Ooencyrtus kuvanae Howard", Phil. Trans. Roy. Soc., B, 229, 275-322 (1938).

17 Thompson, W. R. "Biological Control and the Theories of the Interactions of Populations", Parasitology, 31, 299-388 (1939).

${ }^{18}$ Frisch, K. von, "Psychologie der Bienen", Z. Tierpsych., 1. 9-21 (1937).

"Wood-Jones, F., and Porteus, S. D., "The Matrix of the Mind" (London, 1929).

\title{
STABILITY OF MINERALS IN SEDIMENTARY ROCKS*
}

\author{
By Prof. P. G. H. Boswell, O.B.E., F.R.S.
}

A $\mathrm{S}$ the rocks which form the continental masses are disintegrated by the action of weathering and other geological agents, their detritus accumulates to form sedimentary rocks, ultimately on the sea-bed. In most instances, the constituents of these sedimentary rocks afford a clue to the character of the parent material and furnish information about the probable source and direction of transportation of the detritus (provided, of course, that the investigation is carried out over a sufficiently wide area). In the majority of sediments, especially the coarser types, the bulk of the grains consist, however, of the common mineral quartz, which yields little evidence of a particular source, for it is widely distributed throughout almost all types of rocks. It is able to survive many cycles of erosion because of its high

* Abridged from Part 2 of the presidential address to the Geo. logical Society, March 1941. resistance to chemical alteration and mechanical disintegration. But certain other constituents, usually of rarer occurrence, known as the heavy detrital minerals because of their higher density $(>29)$, are of much greater value as clues to origin, for many of them are characteristic of particular rock-types.

Of the many interesting problems that have arisen from the study of the occurrences of these minerals, that of the relative chemical stability of various detrital species is perhaps the most important and urgent at the present time. Deductions about the origin of sedimentary rocks, based on the varying proportions of constituent minerals, rest on the assumption that their present distribution either does not differ greatly from the original distribution (when the sediments were deposited) or has suffered changes which have affected all the minerals to a similar extent. In 
some cases, at least, such an assumption is unwarranted, for we have good reason to believe that the less stable species gradually decompose in the course of geological time. We find it necessary, therefore, to devise some means of assessing the extent of post-depositional changes in mineral assemblages. Obviously, we can call to our aid experimental work in the laboratory; and the results of such work have shown that minerals vary considerably in their resistance to attack by weak reagents like dilute mineral acids, humic acids, carbon dioxide saturated water and alkalis, although the effects, especially on detrital grains, have not yet been fully investigated. Natural conditions are difficult to imitate, for geological time is not available in the laboratory: we attempt to compensate for this by increasing the concentration of the reagents, but there are obvious objections to such a course. Nevertheless, we can reasonably hope to throw light on the natural processes by making the period of attack as lengthy as is humanly possible ; also, we can try to simulate the field-conditions by adjusting the composition of the reagents to that of various ground-waters, and to imitate the resurgent action of their seasonal rise and fall.

Apart from experimental work of this kind, evidence bearing on the question of the postdepositional stability of detrital minerals can be obtained from field-studies. It is furnished by $(a)$ the comparison of the varying incidence of mineral occurrences throughout the stratigraphical column, especially the comparison of Recent with older sediments, (b) the quantitative study of the various minerals of an assemblage from a particular formation throughout its lateral and vertical extent, and $(c)$ the alteration-phenomena, especially as regards the form and surface features, exhibited by individual mineral grains.

Our experience of the considerable resistance usually offered by rock-forming minerals to solution and our familiarity with the ubiquitous occurrence of detrital minerals may lead us unconsciously to over-emphasize their durability. We may find it a useful corrective, therefore, to bear in mind the significance of two important factors, first, the almost unlimited draft on geological time that is available and, secondly, the relatively large surface area that is open to attack in the case of these minute grains. The work of several investigators, including Tamm, Stevens and Brammall, gives point to this latter consideration, for they found that most of the rock-forming minerals are hygroscopic when finely powdered, and are partially dissociated, the water then giving an alkaline reaction. The importance of these examples of hydrolysis at ordinary temperatures and pressures is manifest, for they suggest that, once such minerals are reduced by natural mechanical action to the size of the clay grade, their decomposition will probably be rapid; and, even in their coarser state, the grains are scarcely likely to escape corrosion under the action of ground-waters.

It is axiomatic to say that, other things being equal, the longer the time available for percolating water to act on minerals, the greater will be the amount of decomposition and the more complete the elimination of vulnerable constituents. Several investigators have directed attention to the fact that the relative abundance of certain minerals (such as andalusite, kyanite, the pyroxenes, the amphiboles, etc.) decreases with the increasing geological age of the sediments. Most striking, in this connexion, is the contrast between the mineral assemblages of the Recent or Pleistocene deposits and those of more ancient sediments. The former assemblages are rich in heavy constituents, both as regards variety and abundance, and the grains are often exceptionally beautiful. The number of species identified from an individual sand may be forty, sixty, or even more. In all, about a hundred and sixty rock-forming minerals have been found in sedimentary rocks, that is, practically as many as are known to occur in the parent crystalline rocks, if we exclude species of great rarity. But about forty of these, including the rarer species, can be found only in geologically youthful deposits. As they occur in ancient crystalline rocks, which have undergone erosion in the normal course of events in past epochs, their absence from the corresponding ancient sedimentary formations must be attributed to subsequent decomposition.

Passing to the consideration of the evidence furnished by the quantitative study of the occurrence of various minerals in a particular sediment, I may recall that, when the character of the mineral assemblage was used as a clue to the provenance of the detrital material, the progressive changes in relative abundance and size of grains of significant minerals, as the deposits were traced laterally, were interpreted as an indication of the relative distance from the source-rocks and thus of the probable direction in which they lay. This deduction followed from the assumption that, as the source became more and more distant, the less stable minerals were gradually eliminated in the reverse order of their resistance to abrasion, fracture and chemical decomposition. But any complacency we might have felt about the correctness of conclusions based on such evidence must have suffered a shock when R. Dana Russell published recently the results of his detailed researches on the lengthy transportation of minerals by the River Mississippi. Here we might have expected to get clear evidence of the gradual disappearance of the less stable elements. However, Russell 
found that the eliminatory effects appear to have been greatly over-estimated, at least with respect to the sand sizes.

The concept that even such 'weak' minerals as the pyroxenes, amphiboles and the felspars are unable to survive more than a few hundred miles of transportation because of abrasion during the journey is quite erroneous. Abrasion produces only minor changes in the mineral composition of Mississippi River sands in 1,100 miles of transport, and even these changes are largely offset by other factors, especially progressive sorting according to size. Similarly, chemical decomposition during transportation also plays but a minor part. The net result is that sands reaching the Gulf of Mexico have nearly the same composition as those at Cairo, Illinois. In order that the general applicability of his conclusions may be tested, Russell pleads for similar investigations of other river deposits, and of beach and dune sands; we shall await these new results with great interest for, although it has been claimed that the gradual elimination alongshore of certain minerals has been established, the investigations have been less intense than Russell's and the area covered in each case was more restricted.

As the challenge thus thrown out must be met we may find it desirable also to re-investigate in at least the same detail those ancient sediments from which deductions have been made about the source of the material. Although, in certain instances, we may have erred on the side of boldness in framing our postulates, I am by no means convinced, after a fresh study of the European literature, that we have depended on them to such an extent that we have in consequence been led into serious error. In those cases where we have relied on the progressive diminution in size of significant detrital minerals, with or without reduction in variety of constituents (and such cases are numerous), and have taken due account of the grading of the sediment, we have doubtless gone but little astray. Also, for some time past, we have been less inclined to be dogmatic in our inferences about the source of detritus, based on records of mineral frequencies, largely because of our conviction that the potency of post-depositional processes should not be underestimated. In those instances where the grade of a sediment remains approximately uniform as the formation is traced laterally, the question of variation of frequency with grain-size obviously does not arise. The same is true if the grading varies, provided that all grades of the sediment have been subjected to mineral analysis. In either of these cases, a progressive change in the abundance of particular minerals may depend on one or both of two factors, distance of transportation and effects of post-depositional processes. Recent work (for example, that of F. Smithson, referred to below) bids fair to enable us to distinguish between the operation of these two factors.

It is a common experience that heavy minerals are often rare in coarse sands, other than natural concentrates, but are plentiful in less coarse sediments down to the clay grade, their distribution in the various grades being determined by their equivalent hydraulic value. As disintegration proceeds and the deposits become finer as a whole, the heavy minerals may be lost to sight because they have passed into the finest grades. The opportunities afforded for investigating all the grades of a present-day sediment are obviously much greater than in the case of a 'fossil' sediment, for portions of the latter have usually become inaccessible as a result of erosion or burial. Our conclusions must then be based on a part only of the deposit, but it has yet to be proved, from studies of Recent deposits, that they are thereby invalidated.

The survival of minerals after they have been incorporated in a sediment clearly depends on the ease or difficulty with which water can percolate through the deposit. Having regard to its slower passage through fine-grained rocks, we might expect such rocks to remain richer mineralogically, were it not that there is the offsetting tendency for clays, because of their mode of origin as the finest products of decomposition of other minerals, to be deficient in mineral variety. The conditions under which the differential effects of percolation are likely to be greatest are those where sands are protected by an impermeable cover. Not only should we then expect that a considerable proportion of the less resistant minerals would be retained in the sands, but also a vulnerable species (such as apatite, for example) would survive in quantity. Actual observations in Great Britain and the United States have shown this to be the case. The contrary state of affairs should be found in sands lying in a basin of clay, with the resurgent effect of ground-waters in full play.

By intensive study of the petrology of a specially chosen sediment, directed to a comparison of the relative abundance of particular minerals in relation to their 'pathology' and the grading of the sediment, light may be thrown on the efficacy of the post-depositional processes. The formation selected for study should preferably have a welldefined and fairly characteristic heavy mineral assemblage, and have been accumulated in such a way that all parts of its outcrop are approximately equidistant from its source-rocks. Even if these conditions are not exactly fulfilled, valuable results can be obtained from the investigation of a suitable sediment, as witnessed by F. Smithson's 
attack on just such a problem recently. Briefly stated, his method has been to study theoretically the variations in proportion shown by the heavy minerals as a result of sorting, of mineralogical changes such as decomposition and reconstitution, and of other processes. He has compared these results with the data obtained from the quantitative investigation of natural deposits. The similarities in the results, which are clearly demonstrated by graphical expression, seem too marked to be fortuitous. From his detailed studies of the Estuarine Series of the Jurassic of Yorkshire, he has been able to prepare maps showing changes in a number of mineral characters in 'contour' form.

Among the features thus represented in Smithson's work are the diminution in the richness of the mineral assemblages, in the percentage of samples containing garnet, in the estimated frequency of garnet and in the actual percentage of the mineral, as the middle of the Yorkshire basin is approached. The result of plotting the frequency of apatite is somewhat similar. On the other hand, the frequency of ilmenite + leucoxene, the frequency of authigenic pyramidal anatase and the percentage of zircon showing outgrowths (all secondary features and doubtless post-depositional) increase regularly towards the middle of the basin. Thus the pattern of the maps suggests that, along the tectonic axis (where the rocks are downfolded), there is an oval belt in which certain processes have been most active, leading to decomposition of garnet, the general impoverishment of the mineral assemblage, and certain secondary changes such as the production of outgrowths and new minerals. If all the mineral characters (including size and relative dimensions of grains) are treated statistically one by one and recorded on maps, an interesting result appears. Had the characteristics been preserved as they were when the sediments were laid down, all the maps should have presented, as it were, a picture of the palæo-geographical conditions prevailing at the time of deposition. Instead, we find that, owing to somewhat localized mineralogical changes, many of the maps present a generalized picture of the tectonics of the area, and in some, doubtless, both pictures are confused. If in some cases, then, impoverishment increases in proximity to the middle of structural basins rather than basins of deposition, we can understand how it was that the frequent coincidence of the two types of basins contributed to the widespread belief that progressive elimination of minerals increased with transport and afforded a clue to the distance and direction of the source.

Turning now to the third line of evidence, that furnished by the form of mineral grains, we recall that faceting, notching and fraying of detritals have frequently been recorded from many ancient sediments. These features have been imitated in the laboratory by Bramlette (who treated garnet with hydrofluoric acid) and Mackie (who subjected various minerals to the action of acid potassium sulphate). Although, as compared with natural processes like the attack by ground-waters, such treatment was drastic, its duration was very short. Naturally corroded grains are especially characteristic of certain stratigraphical horizons, presumably where the environment was exceptionally favourable. The type of corrosion, and therefore the form of the grains, differs according to the properties of the particular mineral, and the general composition of the sediment and the ground-water, although at present the relationship is far from obvious.

The angles, where measurable, of the notching or etching are peculiar to the particular mineral, and are related to the directions of crystal axes, optic axes, cleavage, or other determinable properties. Edelman and Doeglas, after investigating a large number of these corroded grains, are convinced that the features are closely related to the etch-figures of the minerals, where they are known, and are 'relict-structures' marking a stage in the process of gradual elimination of the minerals. The features are certainly post-depositional, for their delicacy and fragility are sueh that they could not possibly have withstood the rough-and-tumble of transportation.

As examples of British stratigraphical formations in which corroded grains are well developed, I may refer to the remarkable etching of garnet in the various sandy horizons of the Carboniferous system, the raggedness of the staurolite in many beds of the Permian and Triassic (in which the garnet is most frequently sub-angular or rounded), the frayed kyanite, saw-edged staurolite and lunate garnet of the Lias - Inferior Oolite sands, and the carious kyanite of the Northampton Ironstone. Numerous other examples could be cited to illustrate the selective character of the corrosion. Probably the phenomenon is related to the composition of the local ground-waters, and its manifestation depends on favourable conditions in the lithology and tectonic arrangement of the rocksuccession, but until such relationships have been investigated in detail at particular localities, any discussion of the manner in which the postdepositional processes operate must be largely conjectural.

P.S. Since the above was written, a paper by M. N. Bramlette has appeared in the Journal of Sedimentary Petrology for April 1941 on "The Stability of Minerals in Sandstone", which contains many new observations and amplifies the above remarks in several important particulars. 